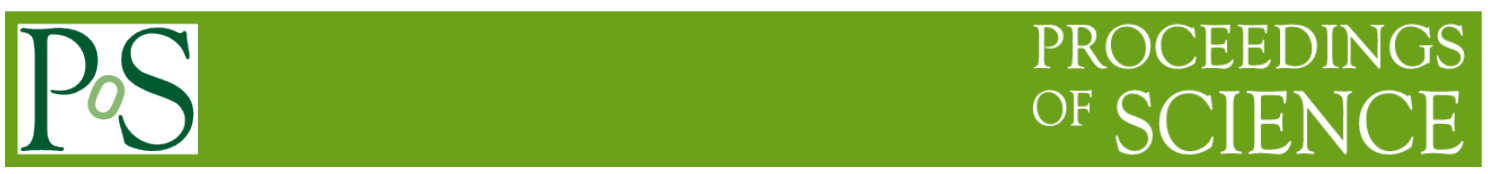

\title{
Cosmic ray detection and Identification at Antarctica in a ground level experiment with NTD
}

\section{Sandhya Dey}

Centre for Astroparticle Physics \& Space Science, Bose Institute

Kolkata 700 091, India

E-mail:dey_s2001@yahoo.com

\section{Rupamoy Bhattacharyya}

Centre for Astroparticle Physics \& Space Science, Bose Institute

Kolkata 700 091, India

E-mail:rupamoy@gmail.com

\section{Sanjay Ghosh}

1Centre for Astroparticle Physics \& Space Science, Bose Institute

2 Departmet of Physics, Bose Institute

Kolkata 700 091, India

E-mail:sanjay@jcbose.ac.in

\section{Atanu Maulik}

Centre for Astroparticle Physics \& Space Science, Bose Institute Kolkata 700 091, India

E-mail:atanu.maulik@gmail.com

\section{Sibaji Raha}

Centre for Astroparticle Physics \& Space Science, Bose Institute Kolkata 700 091, India

E-mail:sibajiraha@gmail.com

\section{Debapriya Syam}

Centre for Astroparticle Physics \& Space Science, Bose Institute Kolkata 700 091, India

E-mail:syam.debapriya@gmail.com 


\begin{abstract}
Searching for Strangelets (small pieces of strongly interacting matter comprising of nearly equal numbers of $u, d$ and $s$ quarks) in cosmic rays, an experiment has been done at Antarctica using passive detectors. We have exposed CR-39 and PET (polyethylene terephthalate sheets) at Maitri and Bharati experimental stations of India. High detection threshold detector PET limits the detection of cosmic rays to relatively high $\mathrm{Z}$ and low energy particles. In this report we will present charge response parameters $\left(V_{t} / V_{b}\right)$ for charged cosmic ray particles observed at ground level. Track parameters viz. diameters, depths and incidence angles of observed charged particles in CR39 show wide distributions. Identification of different groups of particles has been done from the distribution of charge response parameters $\left(\mathrm{V}_{t} / \mathrm{V}_{\mathrm{b}}\right)$ with the help of the calibration curve of $\mathrm{CR}$ 39. Flux of charged particles observed in CR-39 is $\sim 6.2 \times 10-{ }^{4} /\left(\mathrm{cm}^{2} . s e c . s r\right)$ at both the places.
\end{abstract}

36th International Cosmic Ray Conference -ICRC2019-

July 24th - August 1st, 2019

Madison, WI, U.S.A. 


\section{Introduction}

The primary cosmic radiation consists predominantly of protons (90\%), alpha particles (9\%), and 1\% are heavier high energy elements called HZE. Charged particles approaching towards the earth are influenced by various magnetic fields i.e. the galactic, interplanetary, and the geomagnetic fields. Heavy high energy cosmic particles can easily reach the Polar regions as the value of geomagnetic rigidity cut-off is nearly equal to zero there. There is no study found in the literature for detection of heavy cosmic ray particles at ground level at Antarctica. We have used a Nuclear Track Detector having high detection threshold, namely PET, along with CR-39. Passive detectors are suitable for detection of cosmic rays at remote locations as they do not require electrical power or electronic devices. Moreover they also work under adverse weather conditions with good performance and they do not require constant monitoring.

To search for exotic particles like strangelets at mountain altitudes, a high detection threshold nuclear track detector material needs to cut off lighter cosmic rays, particularly the proton background. After trials with several plastic materials, researchers from Bose Institute, Kolkata have found that a particular brand of OHP (over head projector) sheet is a good nuclear track detector. This plastic material goes by the chemical name Polyethylene Terephthalate (PET). Detection threshold of PET is very high; it detected fission fragments but did not detect alpha particles when exposed to $\mathrm{Cf}^{252}$ source [1]. We have optimised the etching conditions and characterization and calibration have been done for this detector using several different ions from accelerators in India and abroad [2-5]. Now PET is a well established passive detector.

In a cosmic ray exposure experiment for a rare event search, this detector automatically cuts off the large part of the cosmic ray background due to its high detection threshold. We have exposed PET and CR-39 at various mountain altitudes at Darjeeling, Ooty and Hanle. A rare event was observed at Darjeeling in PET [6]. A heavy ions with Z $>20$ was observed at Hanle [7]. Cosmic ray background fluxes observed at different mountain altitudes was reported in JCAP04(2017)035[8].

To study the cosmic ray flux of heavy ions in the polar region a stack of solid state nuclear track detectors CR-39 and PET have been exposed at Antarctica. PET films having high track registration threshold $(Z / \beta>140)[2]$ were unable to detect protons and alpha particles. PET detector automatically eliminates the large background of lighter cosmic ray particles. Heavier cosmic particles- Carbon onwards, could be detected by PET, but detectability (with reference to energy) is limited by its high detection threshold where as CR-39 detects protons having energy $\mathrm{E}<13 \mathrm{MeV}$ and alpha particles with $\mathrm{E}<210 \mathrm{MeV}$ and heavier ions with correspondingly higher energies.

\section{Exposure at Antarctica}

One stack of three A4 size PET sheets with a small piece $(6.5 \times 3.5) \mathrm{cm}^{2}$ of CR-39 on it was exposed at Maitri station at Antarctica. The period of exposure was March $10^{\text {th }} 2015$ to March $17^{\text {th }} 2015$ and total duration of exposure was $5.796 \times 10^{5}$ sec. Detectors were placed horizontally on a wooden platform on the roof of an abandoned construction. Exposure was also made at Bharati station. Exposure period was January $7^{\text {th }} 2015$ to January $18^{\text {th }} 2015$ to a stack of three A4 size PET films with a small piece of CR-39 plate placed on it and total duration was $9.357 \times 10^{5}$ 
sec. Fig.1 shows the photograph of the detector set up at Maitri station. Exposures have been done by the scientific group of $34^{\text {th }}$ Indian Scientific Expedition to Antarctica (34-ISEA). Locations of Bharati station and Maitri station are shown in fig 2.

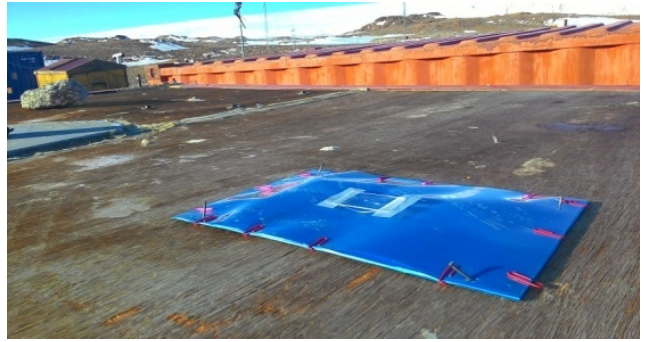

Fig.1. Detectors placed at Maitri station

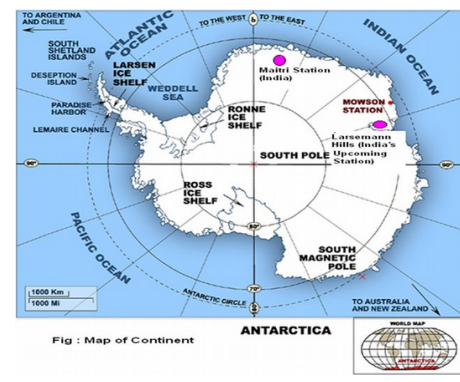

Fig.2. Maitri and Bharati station in the map

\section{Etching and Scanning}

The exposed films were etched in an aqueous solution of $\mathrm{NaOH}$ of concentration $6.25 \mathrm{~N}$. The etching temperature for CR-39 was $70^{\circ} \mathrm{C}$ and for PET, $55^{\circ} \mathrm{C}$. Etching durations were 3 hours for PET and 6 hours for CR-39. We have scanned these films under the dry objective of a Leica DMR4000B microscope connected with a computer loaded with automated image analysis software. Images of tracks in CR-39 are shown in fig 3a \& fig.3b. Image of PET films seen under the dry objective of microscope are shown in fig.3c.Track density has been counted and track parameters i.e. track diameters and depths and incidence angles (angle with the vertical) have been measured for both of the CR-39 plates exposed at Maitri and Bharati.

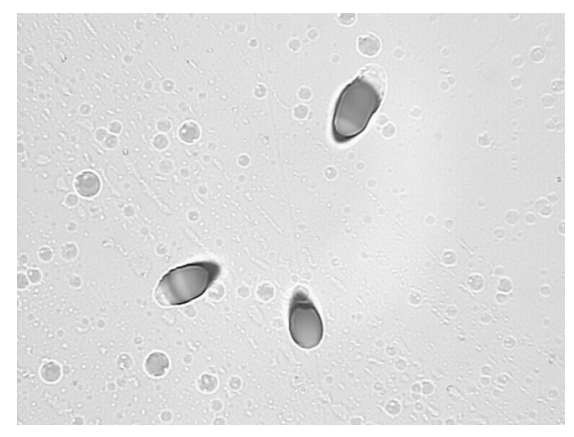

Fig.3a.Tracks observed in CR-39 at Maitri station

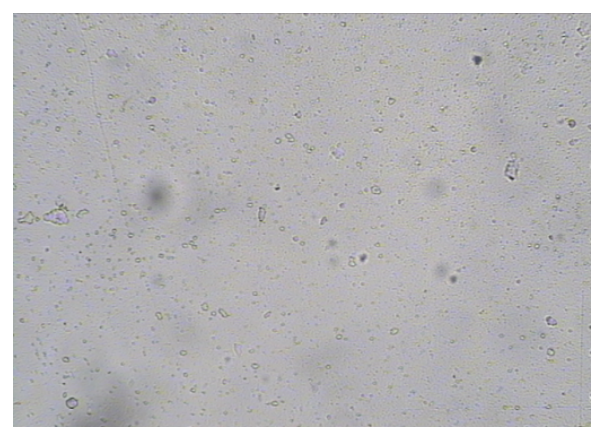

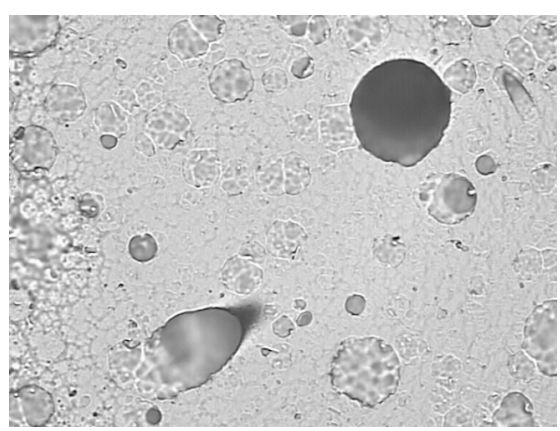

Fig 3b.Tracks observed in CR-39 at Bharati station

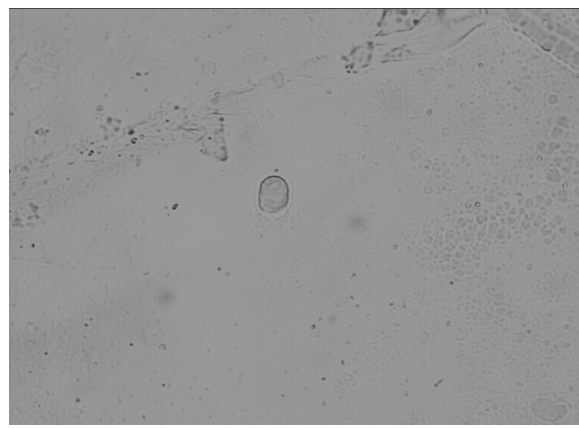

Fig.3c.Microphotographs of PET at Maitri (left) and Bharati (right) [Image size of fig.3a \&fig.3c are $234 \mu x 170 \mu$ \& fig. $3 b$ is $117 \mu \times 85 \mu$ ] 


\section{Observation and analysis}

The flux of heavy ions is $<2.2 \times 10^{-8} /\left(\mathrm{cm}^{2}\right.$.s.sr $)$ in PET. Track registration threshold of PET $(\mathrm{Z} / \beta \approx 140)$ is much higher than that of $\mathrm{CR}-39(\mathrm{Z} / \beta \approx 6)[1,2]$. Observations at various mountain altitudes established that PET rejects the large low-Z cosmic ray background including the alpha particles from radon decay [3]. Charged particle flux observed in CR-39 at Maitri station is $6.18 \times 10^{-4} /\left(\mathrm{cm}^{2} . \mathrm{s} . \mathrm{sr}\right)$ and at Bharati station it is $6.21 \times 10^{-4} /\left(\mathrm{cm}^{2} . \mathrm{s} . \mathrm{sr}\right)$. Preliminary results were reported by us in the $27^{\text {th }}$ SSNTD conference [9].

The minor axis diameters of tracks observed in CR-39 at both stations are shown in fig.4a and fig.4b Charge response parameter $\mathrm{V}_{\mathrm{t}} / \mathrm{V}_{\mathrm{b}}$ from the measurement of track parameters are shown in fig.5a and fig.5b for CR-39 plates.

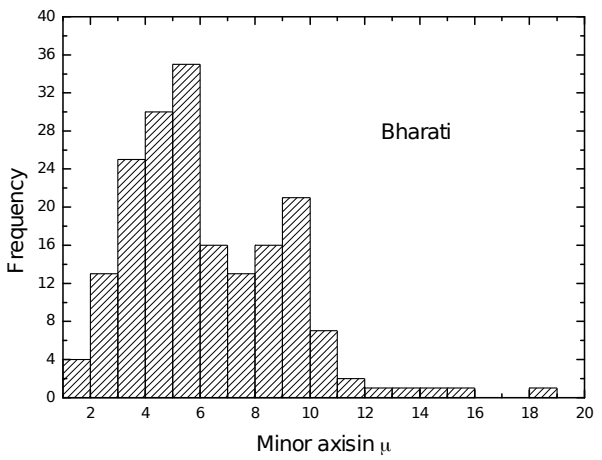

Fig.4a

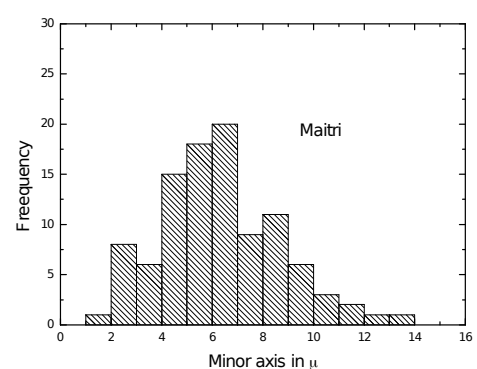

Fig.4b

Track registration threshold for SSNTD is the lowest $Z / \beta$ of impinging ion, where $Z$ is the atomic number of ion and $\beta=v / c, v$ is velocity of ion and $c$ is the velocity of light for which a track is formed. Therefore highest energy for which track will be formed in a particular NTD will be restricted by $Z / \beta$. For CR-39 the threshold is $>6$ and for PET it is $>140$. Therefore an ion has an energy limit above which it will not produce any track in a NTD, and below which it will produce a track. Energy will be different for different ions i.e. for different $Z$ as $(Z / \beta)_{\text {threshold }}$ is fixed. Energies for different ions at $Z / \beta$ threshold are shown fig. 6 for both CR-39 and PET. Therefore it is very unusual to find any track of high energy cosmic rays in PET. 


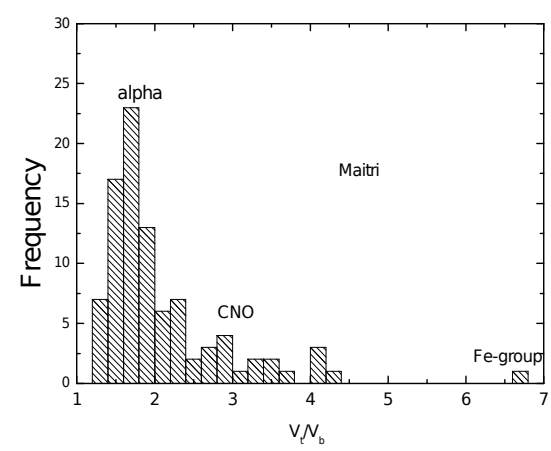

Fig.5a

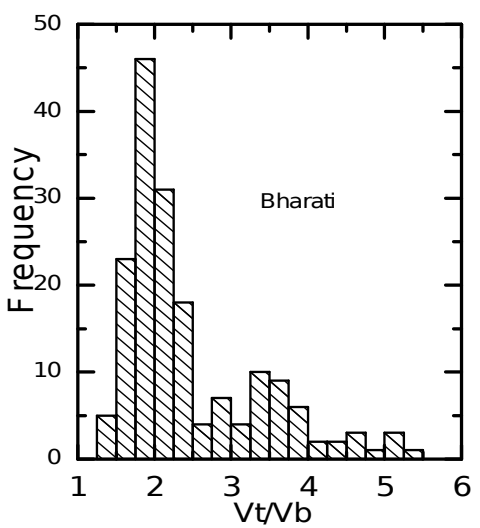

Fig.5b

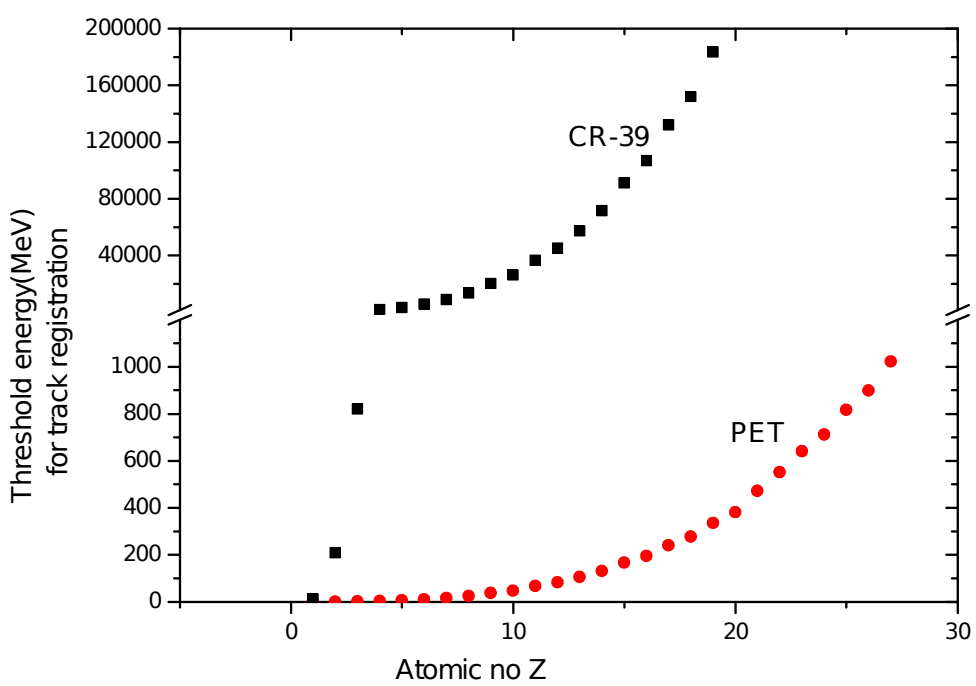

Fig.6. Track registration threshold of CR-39 \& PET

\section{Conclusion}

We have not found any tracks in PET but many tracks in CR-39 after short duration exposure. We can conclude that observation of a track of any high energy event in PET at Antarctica may be claimed as an unusual event.

\section{Acknowledgement}

The work is funded by IRHPA (IR/S2/PF-01/2011 dated 26.06.2012) of the Science and Engineering Research Council (SERC), DST, Government of India, New Delhi.We would like toacknowledge with thanks the team members of 34-ISEA for exposure of plates. 


\section{References}

[1] B. Basu, S. Banik, A. Mazumder, S. Raha, S .Saha, S. K. Saha, Indian Journal of Physics 79(3), 279-283(2005).

[2] Characterization and calibration of SSNTD for heavy-ion detection and strangelet search in cosmic rays, D. Bhowmik S. Dey, A. Maulik, S. Raha, S. Saha, Swapan K. Saha, D. Syam, Nucl. Inst. \& Meth. B (2011)269 pp 197 - 201.

[3] Calibration of a salid state nuclear track detector (SSNTD) with high detection threshold to search for rare events in cosmic rays, S. Dey, D. Gupta, A. Maulik, Sibaji Raha, Swapan K.Saha, D. Syam, J. Pakarinen, D. Voulot, F. Wenander,Astroparticle Physics 34 (2011) $805-808$

[4] Bhattacharyya, R., Dey, S., Maulik, A., Ghosh, S.K., Raha, S., Syam, D.,Nucl. Instr. and Meth. B 370 (2016) 63

[5] Basu , B. , Dey, S., Fischer, B., Maulik, A., Mazumder, A., Raha, S., Saha ,S., Saha, S.K., Syam, D., Radiation measurements 43(2008) S95-S97

[6] Observation of a rare cosmic ray event at mountain altitude, Basudhara Basu, Sibaji Raha, Swapan k Saha, Sukumar Biswas, Sandhya Dey, Atanu Maulik, Amal Mazumdar ,Satyajit Saha, Debapriyo Shyam, Astroparticle Physics 61(2015)88-92

[7] Detection of Heavy Cosmic Ray Particle ( $\mathrm{Z}>20)$ at Hanle, Ladakh at an altitude of 4.5 km a.s.l. using SSNTD- Sandhya Dey,Atanu Maulik, Sibaji Raha, Swapan K. Saha, andDebapriyo Syam, Procedings 32ND International Cosmic Ray Conference, BeiJing 2011.

[8] Bhattacharyya, R., Dey, S., Maulik, A., Ghosh, S.K., Raha, S., Syam, D., Journal of Cosmology and Astrophysics, Vol 04, April 2017

[9] Investigation of heavy charged particle flux in cosmic rays at Antarctica with high detection threshold PET films, A Maulik ,S Dey, R. Bhattacharyya, S K Ghosh, S Raha, and D SYam POSTER ICNTRM 2017-23.8.17 